Malcolm Lemyze Jihad Mallat

\title{
Understanding negative pressure pulmonary edema
}

M. Lemyze (®) · J. Mallat

Department of Respiratory and Critical Care

Medicine, Schaffner Hospital, 99 route de la

Bassée, 62300 Lens, France

e-mail: malcolmlemyze@yahoo.fr

\section{J. Mallat}

Department of Anesthesiology, Schaffner

Hospital, Lens, France

\author{
Abbreviations \\ NPPE Negative \\ pulmonary edema \\ PE Pulmonary edema \\ UAO Upper airway obstruction \\ ICU Intensive care unit \\ ARDS Acute respiratory distress \\ syndrome \\ NIV Noninvasive positive \\ pressure ventilation
}

Electronic supplementary material

The online version of this article (doi:10.1007/s00134-014-3307-7) contains supplementary material, which is available to authorized users.
Negative pressure pulmonary edema (NPPE) is a form of noncardiogenic pulmonary edema (PE) that results from the generation of high negative intrathoracic pressure (NIP) needed to overcome upper airway obstruction (UAO). NPPE is a potentially life-threatening complication that develops rapidly after UAO in otherwise healthy young persons who are capable of producing large markedly NIPs. The incidence of NPPE, in patients developing acute UAO, has been estimated to be up to $12 \%$ [1]. The true incidence, however, is not known and may be higher than has been suggested, since many cases may have been misdiagnosed because of a lack of familiarity with the syndrome. All causes of obstructed upper airway may lead to NPPE [2]. However, the most commonly reported etiology of NPPE in adults is laryngospasm during intubation or in the postoperative period after anesthesia (50\% of cases of NPPE) [3]. Nevertheless, NPPE may be more common in ICU patients than is thought; For instance, ventilation with low tidal volume during the acute phase of ARDS in patients with increased respiratory drive can lead to patient-ventilator asynchrony that causes increased breathing effort and the generation of high NIPs that will further worsen PE. Also, strong inspiratory efforts in the presence of increased resistive work of breathing will lead to negative alveolar pressures mimicking the cardiothoracic relationships present during NPPE, and may contribute to extubation failure in some patients.

Understanding the pulmonary fluid homeostasis is crucial to comprehend the mechanisms responsible for pulmonary edema formation. In the normal lung, the net fluid transfer across the pulmonary capillaries depends on the net difference between hydrostatic and colloid osmotic pressures, as well as on the permeability of the capillary membrane (Starling's law). Under normal conditions, most of this filtered fluid is removed from the interstitium through the lymphatic system to return to the systemic circulation [4]. The alveolar epithelium, because of its tight intercellular junctions, acts as an effective barrier limiting water intrusion into alveolar spaces. However, when the hydrostatic pressure in the pulmonary capillary bed increases and/or the lung interstitial pressure decreases, the rate of transvascular fluid filtration rises, causing edema in the perimicrovascular interstitial spaces, 


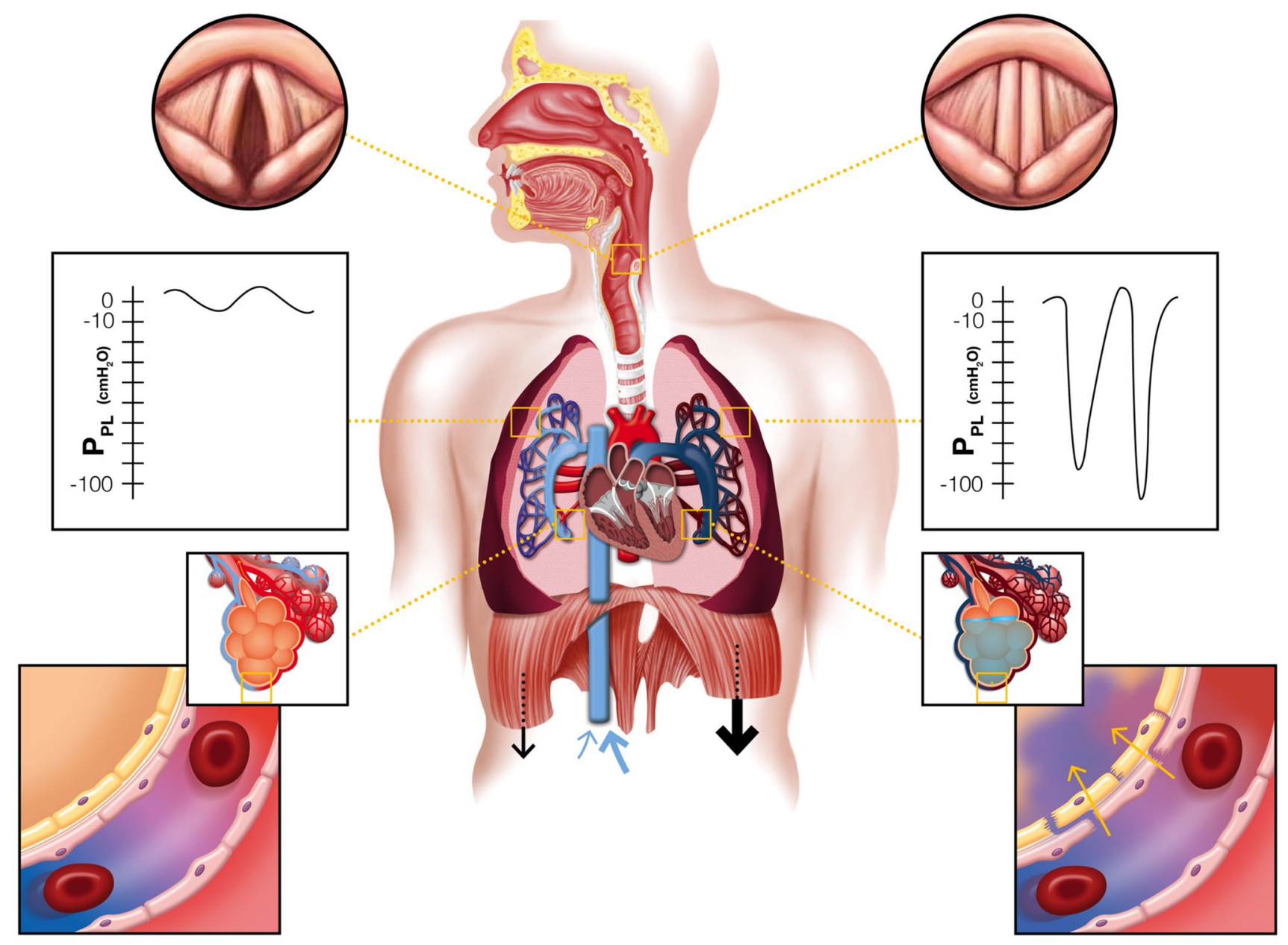

Fig. 1 As shown in the left part of the illustration, breathing through the normally open upper airway requires minimal diaphragmatic efforts (thin black arrow) that generate small levels $\left(-2\right.$ to $\left.-8 \mathrm{cmH}_{2} \mathrm{O}\right)$ of negative pleural pressure $\left(P_{\mathrm{PL}}\right)$ during inspiration. In normal conditions, the alveolar-capillary pressure gradient is small, and when hydrostatic pressures slightly increase in the pulmonary capillary bed, the fluid overload may be offset by increased lymphatic drainage. Conversely, inspiration against an obstructed upper airway-as represented by closed vocal cords in the right side of the illustration-requires forceful diaphragmatic efforts (large black arrow) generating high levels ( -50 to $140 \mathrm{cmH}_{2} \mathrm{O}$ ) of negative $P_{\mathrm{PL}}$ that increase venous return to the right side of the heart (large blue arrow). This may result in higher hydrostatic pressures in the pulmonary capillaries and a sudden drop of pressures in the alveolar spaces, creating a huge pressure gradient across the pulmonary capillary wall and disruption of the alveolar-capillary membrane, leading to alveolar flooding and pulmonary edema (yellow arrows) and maybe alveolar flooding if a critical quantity of edema fluid in the interstitial space has been reached [4, 5].

What is the pathophysiology of NPPE? Two different mechanisms have been suggested to explain the pathogenesis of PE during UAO. One belief is that NPPE is developed by substantial fluid shifts due to swings in intrathoracic pressure [6]. Markedly NIP is generated by deep inspiratory efforts against an occluded airway or a closed glottis (modified Müller maneuver). Young healthy subjects can generate very high levels of negative inspiratory pressure with a maximum of $-140 \mathrm{cmH}_{2} \mathrm{O}$ [7]. This NIP is transmitted by the same amount to the intrapleural spaces, resulting in augmentation of venous return to the right side of the heart, and pulmonary venous pressures, while decreasing perivascular interstitial hydrostatic pressure, which favors movement of fluid from the pulmonary capillaries into the interstitium and alveolar spaces [8] (Fig. 1). Also, the hyperadrenergic state associated with catastrophic UAO can cause peripheral vasoconstriction and an increase in venous return, which could further increase pulmonary blood flow, contributing to edema. Furthermore, the NIP increases left ventricular afterload by increasing the transmural left ventricular pressure, thus raising ventricular wall tension [9]. The increase in afterload depresses left ventricular ejection. In addition, the resulting hypoxemia decreases myocardial contractility and increases 
pulmonary arterial resistance. The fall in left ventricular ejection fraction augments in succession end-diastolic pressure, left atrial pressure, and pulmonary venous pressure, further increasing the pulmonary capillary hydrostatic pressures that promote the formation of PE [9]. Therefore, the combination of increased preload and afterload associated with a decrease in pulmonary interstitial pressure cause a high increase in the hydrostatic pulmonary pressure gradient (disturbance of the Starling equilibrium), allowing transudation of fluid out of the pulmonary capillary into the lung interstitium, resulting in PE. This mechanism of NPPE is similar to hydrostatic PE as observed in patients suffering from congestive heart failure or volume overload states.

The second suggested mechanism is that the mechanical stress developed from respiration against an obstructed upper airway may induce breaks in the alveolar epithelial and pulmonary microvascular membranes, resulting in increased pulmonary capillary permeability and protein-rich PE $[7,10]$. This theory is based on the concept of wall stress failure developed more than 20 years ago by West et al. [11], in which increasing transmural pulmonary capillary pressures cause disruption of the alveolar-capillary membrane with resultant high-permeability PE. In animals, when pulmonary capillaries are subjected to increased transmural pressure, ultrastructural damage of the walls of the capillaries and alveolar epithelium is observed under scanning electron microscope [12]. Stress failure of pulmonary capillaries has been suggested to be involved in several conditions causing PE and hemorrhage, including neurogenic and high-altitude PE [13], and following intense exercise in elite human athletes [14]. This indicates that acute increases in transmural pulmonary capillary pressures as observed in NPPE may lead to high-permeability PE [15]. However, Fremont et al. [2], in 10 NPPE patients, found a low PE fluid-to-serum protein ratio with normal alveolar fluid clearance, further supporting a hydrostatic mechanism for edema fluid formation. Nevertheless, we believe that the pathogenesis of NPPE is probably multifactorial, ranging from transudative to high-permeability edema when a very high transmural pulmonary capillary pressure has been produced.

Treatment of NPPE generally includes maintaining a patent airway, and oxygen supplementation with addition of positive end-expiratory pressure or noninvasive positive pressure ventilation (NIV) as guided by physical examination and arterial blood gas analysis. Mechanical ventilation should be reserved for severe patients who do not respond to NIV. Diuretics are often used; however, there is no evidence of their utility, and they may exacerbate hypovolemia and hypoperfusion. Ultimately, NPPE usually has a rapidly resolving clinical course in $12-48 \mathrm{~h}$ when recognized early and treated immediately.

Understanding the pathophysiological mechanisms contributing to PE can help in distinguishing NPPE from other causes of noncardiogenic PE, thus preventing use of inappropriate and dangerous treatment for patients with NPPE.

Open Access This article is distributed under the terms of the Creative Commons Attribution Noncommercial License which permits any noncommercial use, distribution, and reproduction in any medium, provided the original author(s) and the source are credited.

\section{References}

1. Tami TA, Chu F, Wildes TO, Kaplan M (1986) Pulmonary edema and acute upper airway obstruction. Laryngoscope 96:506-509

2. Fremont RD, Kallet RH, Matthay MA, Ware LB (2007) Postobstructive pulmonary edema: a case for hydrostatic mechanisms. Chest 131:1742-1746

3. Louis PJ, Fernandes R (2002) Negative pressure pulmonary edema. Oral Surg Oral Med Oral Pathol Oral Radiol Endod 93:4-6

4. Matthay MA, Folkesson HG, Clerici C (2002) Lung epithelial fluid transport and the resolution of pulmonary edema. Physiol Rev 82:569-600

5. Ware LB, Matthay MA (2005) Clinical practice. Acute pulmonary edema. N Engl J Med 353:2788-2796
6. Oswalt CE, Gates GA, Holmstrom MG (1977) Pulmonary edema as a complication of acute airway obstruction. JAMA 238:1833-1835

7. Cook CD, Mead J (1960) Maximum and minimum airway pressures at various lung volumes in normal children and adults. Fed Proc 19:377

8. Krodel DJ, Bittner EA, Abdulnour R, Brown R, Eikermann M (2010) Case scenario: acute postoperative negative pressure pulmonary edema. Anesthesiology 113:200-207
9. Buda AJ, Pinsky MR, Ingels NB Jr, Daughters GT 2nd, Stinson EB, Alderman EL (1979) Effect of intrathoracic pressure on left ventricular performance. N Engl J Med 301:453-459

10. Koh MS, Hsu AA, Eng P (2003) Negative pressure pulmonary oedema in the medical intensive care unit. Intensive Care Med 29:1601-1604

11. West JB, Mathieu-Costello O (1992) Stress failure of pulmonary capillaries: role in lung and heart disease. Lancet 340:762-767

12. West JB, Tsukimoto K, MathieuCostello O, Prediletto R (1991) Stress failure in pulmonary capillaries. J Appl Physiol 70:1731-1742 
13. West JB, Colice GL, Lee YJ, Namba Y, 14. Hopkins SR, Schoene RB, Henderson Kurdak SS, Fu Z, Ou LC, MathieuCostello O (1995) Pathogenesis of highaltitude pulmonary oedema: direct evidence of stress failure of pulmonary capillaries. Eur Respir J 8:523-529 WR, Spragg RG, Martin TR, West JB (1997) Intense exercise impairs the integrity of the pulmonary blood-gas barrier in elite athletes. Am J Respir Crit Care Med 155:1090-1094
15. Dicpinigaitis PV, Mehta DC (1995)

Postobstructive pulmonary edema induced by endotracheal tube occlusion. Intensive Care Med 21:1048-1050 